\title{
Identification of the Mechanical Properties of the Coating Using the Digital Image Correlation Technique
}

\author{
Sebastian Lubowicki, Jakub Augustyniak, Dariusz M. Perkowski \\ Department of Mechanics and Applied Computer Science, Faculty of Mechanical Engineering, \\ Bialystok University of Technology, 45C Wiejska Str., 15-351 Bialystok, Poland \\ s.lubowicki@doktoranci.pb.edu.pl, j.augustyniak@pb.edu.pl, d.perkowski@pb.edu.pl
}

\section{Extended Abstract}

The aim of this paper was to develop a method for determining mechanical properties based on the example of the Young's modulus of aluminium specimens coated by hard-anodizing method. The methodology was based on inverse elasticity theory correlated with displacement field measurements carried out by high speed photography as data for digital image correlation (DIC) technique.

Eight specimens with dimensions $15 \mathrm{~mm} \times 2,1 \mathrm{~mm} \times 130 \mathrm{~mm}$ were prepared for the test. Four of them were hard-anodized, and the rest of them were aluminium without coating. The oxide coating layer had thickness equal to $0,05 \mathrm{~mm}$, and were made by type II anodizing. The research was conducted in two ways. The first method consisted in determining the Young's modulus on the basis of a well-known method using vibrations of a cantilever beam for two cases, i.e. a cantilever beam with or without an anodizing layer. The idea of the test was to force the specimens to vibration through a modal hammer and record the vibration until it is stabilized. The process was recorded with ultra-high frequency, for period of time set to three seconds. Second tests was unidirectional tensile test by using Shimadzu AGX-V testing machine. The velocity of tensile tests were set to $0,08 \mathrm{~mm} / \mathrm{s}$ and recorded with two compartments of frequency by 3D Aramis system. First one was equal to $25 \mathrm{~Hz}$ and recorded for 600 images to perfectly capture linear elasticity of material, second one was set to $1 \mathrm{~Hz}$ and performed until specimen rupture.

The next step was to analyze received recordings by using GOM Correlate system and digital image correlation software by tracking movement of markers made on the specimen surface. This process allowed to determine the displacement distributions on every plane of individual specimens. In the uniaxial tensile test, the strain as a function of time was determined and subsequently correlated with force value obtained from tensile strength testing machine. To correlate those data interpolating algorithm was created. In case of vibration analysis, the vibration characteristics of individual specimens were determined on the basis of the Fourier analysis and cleaned of environmental noise.

Afterwards, the obtained empirically data were applied to numerical simulation for two cases of test, i.e. a cantilever beam and tensile test. The inverse problem of the elasticity theory was applied to build numerical model, which determines the value of the Young's modulus of individual materials based on the assumption that the mechanical properties of the substrate are known. The formulated problem was solved by using COMSOL Multiphysics software.

The results obtained from both methods were compared and conclusions on the applicability of the proposed method were created.

Acknowledgements: The research has been conducted as part of a project at the Faculty of Mechanical Engineering of the Bialystok University of Technology. Project number WZ/WM-IIM/3/2020. 